\title{
The Generalized Pythagorean Comma Harmonic Powers of a Fundamental Frequency Are Equivalent the Standing Wave Harmonic Fraction System
}

\author{
Donald Chakeres \\ The Ohio State University, Columbus, USA \\ Email: Donald.chakeres@osumc.edu
}

How to cite this paper: Chakeres, D. (2018) The Generalized Pythagorean Comma Harmonic Powers of a Fundamental Frequency Are Equivalent the Standing Wave Harmonic Fraction System. Advances in Pure Mathematics, 8, 652-665. https://doi.org/10.4236/apm.2018.87038

Received: June 1, 2018

Accepted: July 16, 2018

Published: July 19, 2018

Copyright $\odot 2018$ by author and Scientific Research Publishing Inc. This work is licensed under the Creative Commons Attribution International License (CC BY 4.0).

http://creativecommons.org/licenses/by/4.0/ (c) (i) Open Access

\begin{abstract}
Purpose: The Pythagorean Comma refers to an ancient Greek musical, mathematical tuning method that defines an integer ratio of exponential coupling constant harmonic law of two frequencies and a virtual frequency. A Comma represents a physical harmonic system that is readily observable and can be mathematically simulated. The virtual harmonic is essential and indirectly measurable. The Pythagorean Comma relates to two discrete frequencies but can be generalized to any including infinite harmonics of a fundamental frequency, $v_{\mathrm{F}}$. These power laws encode the physical and mathematical properties of their coupling constant ratio, natural resonance, the maximal resonance of the powers of the frequencies, wave interference, and the beat. The hypothesis is that the Pythagorean power fractions of a fundamental frequency, $V_{\mathrm{F}}$ are structured by the same harmonic fraction system seen with standing waves. Methods: The Pythagorean Comma refers to the ratio of $(3 / 2)^{12}$ and $2^{7}$ that is nearly equal to 1 . A Comma is related to the physical setting of the maximum resonance of the powers of two frequencies. The powers and the virtual frequency are derived simulating the physical environment utilizing the Buckingham $\Pi$ theorem, array analysis, and dimensional analysis. The powers and the virtual frequency can be generalized to any two frequencies. The maximum resonance occurs when their dimensionless ratio closest to 1 and the virtual harmonic closest to $1 \mathrm{~Hz}$. The Pythagorean possible power arrays for a $v_{\mathrm{F}}$ system or any two different frequencies are evaluated. Results: The generalized Pythagorean harmonic power law for any two different frequencies coupling constant are derived with a form of an infinite number of powers defining a constant power ratio and a single virtual harmonic frequency. This power system has periodic and fractal properties. The Pythago-
\end{abstract}


rean power law also encodes the ratio of logs of the frequencies. These must equal or nearly equal the power ratio. When all of the harmonics are powers of a $V_{\mathrm{F}}$ the Pythagorean powers are defined by a consecutive integer series structured in the identical form as standard harmonic fractions. The ratio of the powers is rational, and all of the virtual harmonics are $1 \mathrm{~Hz}$. Conclusion: The Pythagorean Comma power law method can be generalized. This is a new isomorphic wave perspective that encompasses all harmonic systems, but with an infinite number of possible powers. It is important since there is new information: powers, power ratio, and a virtual frequency. The Pythagorean relationships are different, yet an isomorphic perspective where the powers demonstrate harmonic patterns. The coupling constants of a $v_{\mathrm{F}}$ Pythagorean power law system are related to the $v_{F} s$ raised to the harmonic fraction series which accounts for the parallel organization to the standing wave system. This new perspective accurately defines an alternate valid physical harmonic system.

\section{Keywords}

Power Laws, Harmonic Systems, Standing Wave, Harmonic Fractions,

Dimensional Analysis, Buckingham Pi Theorem, Pythagorean Comma

\section{Introduction}

\subsection{The Ancient Greeks Insights into the Universal Properties of Waves and Harmonic Systems}

Pythagoras is best known for the fundamental relation in Euclidean geometry among the three sides of a right triangle. He is less well known as the father of the mathematics and physics of all harmonic and wave systems, including music. The western musical scale is referred to as the Pythagorean musical scale [1] [2] [3]. Many other ancient Greeks, including Euclid, expanded the understanding of harmony, sinusoidal waves, periodic functions, and wave interactions through their study of music. They recognized that harmony was defined by the most resonant possibilities of a fundamental frequency and wavelength. Though their focus was related to music, their observations are the foundation of the understanding of all wave properties and interactions independent of their physical or mathematical setting. The Greeks described the frequency and reciprocal wavelength relationship, overtone series, harmonic fractions, partial harmonic fractions, frequency coupling constants, and the integer-based harmonic power law relationships. A power law is a functional relationship between two quantities, where one quantity varies as a power of another. Kepler's Laws immediately come to mind.

The Greeks believed that the most harmonic of musical frequencies were related to ratios and powers of the first 4 integer harmonic overtones, $\{1,2,3,4\}$. These ratios include $2,3^{2} / 2^{3}, 2^{2} / 3,3 / 2,2^{2}$ corresponding respectively to the fundamental frequency, $V_{F}$ second, fourth, fifth, and octave notes. The Greeks central doctrine was that "all nature consists of harmony arising out of numbers" [4]. 
In music theory, an interval is a separation between two sonic frequencies. An interval mathematically represents the coupling constant ratio of two frequencies. Successive power coupling constant intervals of a musical frequency result in an exponential change of frequency, even though the human ear perceives this as a linear change in pitch. Musical intervals are often defined as cents, a unit derived from the logarithm base 2 of the frequency ratio. A minute interval is described as a Comma, a result of the tuning for the same note utilizing two different mathematical and physical methods. The musical Commas closely approximate unity since the two frequencies are nearly identical.

These ancient mathematical and physical harmonic properties define universal wave properties and interaction phenomena. We decided to explore the generalized Pythagorean Comma harmonic power law application to any harmonic system including one based on a fundamental frequency, $v_{\mathrm{F}}$.

\subsection{The Pythagorean Comma Power Law}

The Greeks observed that the next most harmonic musical frequency in relationship to 2 , the octave, is the number 3 , or, the "fifth" note. They observed that the fraction, (3/2) which is the fifth within a single octave, raised to the 12th power, equaled 129.746 , and 2 raised to the seventh power equaled 128 . The ratio of these two is then 1.01364 . This is the closest possible power ratio to unity. This ratio must be irrational. This ratio is equivalent to the fifth interval raised 12 times is near equals to the octave raised 7 times. Physically and mathematically those frequencies and notes are the most resonant of all of the note possibilities. Equivalently, the $12^{\text {th }}$ power of $3,531,441$, is nearly equal to that of the $19^{\text {th }}$ power of 2, 524,288, Equation (1). In musical scale and physical terms, the $12^{\text {th }}$ "fifth" is maximally resonant, with the $19^{\text {th }}$ octave. This relationship is described in music theory as the circle of "fifths" since the pattern repeats [1] [2]. These power harmonic relationships are periodic and have fractal properties. A fractal is a repeating pattern that continues independently of scale, and where each part of which has the same statistical character as the whole [5]. The dimensionless resonance ratio, of these two frequencies is referred to as the Pythagorean Comma. Due to the primality of both 2 and 3, no integral power of 2 equates to a power of 3 .

$$
\left(\frac{3^{12}}{2^{19}}\right)=\left(\frac{(3 / 2)^{12}}{2^{7}}\right)=1.01364326
$$

It is essential in the physics, mathematics, and the tuning of musical instruments. The resonance of the two Pythagorean power scale frequencies is very close, but not perfect. “... thus a perfectly pure harmonious system of tones is impossible, not only physically but arithmetically. The numbers themselves, by which the tones can be expressed, have insoluble irrationalities" [6].

If a harmonic system is composed solely of powers of the $V_{F}$, then the virtual harmonics must be equal to $1 \mathrm{~Hz}$, and there are no irrational power harmonic 
frequencies. Physically, these irrationalities in music are related to virtual frequencies leading to an audible beat. These minute frequency intervals are interpreted as dissonances and are essential in tuning musical instruments with the goal of the most pleasing harmonic result. These ancient mathematical and physical harmonic properties define universal wave interaction phenomena just as valid as the beat to define wave interference.

The Pythagorean Comma power perspective represents a real physical system that is observable, essential, indirectly measurable through the beat, and can be computationally simulated. The virtual harmonic is necessary to maintain the physical units and mathematically when the two frequencies are not powers of each other. A simple example would be a sound generator system where the frequencies are 8 and $4 \mathrm{~Hz}$. The powers of the frequencies would be gradually and systematically stepped through. Every other power of the $8 \mathrm{~Hz}$ would be perfectly resonant with every third power $4 \mathrm{~Hz}$ wave. This pattern would generate an infinitely repeating series of sounds with a resonance that would be readily observable. Since the virtual power harmonic, $v_{P h}$, of that system would be $1 \mathrm{~Hz}$, it is indirectly measured based on perfect resonance. If the frequencies were an irrational frequency of $8.081273 \mathrm{~Hz}$ and rational $4 \mathrm{~Hz}$ instead, then the maximum resonance would be associated with a beat of $0.6534867 \mathrm{~Hz}$ which is measurable. The virtual power harmonic could be derived.

\subsection{Hypothesis}

The hypothesis includes that the Pythagorean Comma power law method can be generalized for any harmonic system and wave interaction. Important mathematical, harmonic, and physical insights from this method that are not recognizable using standard harmonic methods that include, the powers, ratio of the powers and the virtual harmonic. The organization of the Pythagorean power laws of fundamental frequency, $V_{F}$ are structured in the fraction system identical to standing wave harmonic fractions, but as powers. All harmonic systems based on a $V_{F}$ have both an infinite number of harmonics based on the overtone series, and another infinite set of powers linked to a single virtual harmonic of $1 \mathrm{~Hz}$ in the Pythagorean power system.

\subsection{Spontaneous Harmonic Spectra, Power Laws, and Harmonic Systems}

Spontaneous natural harmonic systems typically demonstrate spectral properties of an infinite number of possibilities mathematically related to the consecutive integer harmonic fraction series, $1 / n$, such as the overtone series of a vibrating string. These harmonics are the greatest possible resonance of the harmonic and the $V_{F}$. The possible wave amplitudes or energies approach zero, so not all of them are observable as the integer value increases, but essential to the mathematical system. A classic standing wave example is a vibrating string has a fundamental frequency, $V_{F}$. There are observable overtones, harmonics, of the product of $v_{F}$ with the consecutive integer series. The harmonic wavelengths are 
related to the harmonic fraction coupling constant series $\{1,1 / 2,1 / 3,1 / 4 \ldots\}$ times the fundamental wavelength. The remaining portion of the harmonic oscillator follows the partial harmonic fraction series of $\{2 / 3,3 / 4,4 / 5 \ldots\}$ wavelengths and frequencies of $\{3 / 2,4 / 3,5 / 4 \ldots\}$. Partial harmonic fractions are related to $1 \pm(1 / n)$. Table 1 is an integer fraction array of a standard harmonic system. The coupling constants that transform one harmonic to another do not change based on the $V_{F}$ An open tube instrument and a laser demonstrate a similar pattern, but only the product of $V_{F}$ and the odd numbers of the consecutive series [1] [7]. The smaller integers are those harmonics that are physically dominant, most observable.

\subsection{Wave Interaction, Superposition, Interference, and the Beat Phenomenon}

There are many wave interactions, one is a beat frequency, which is related to

Table 1. The harmonic integer fraction system of a fundamental frequency and standing waves. This table is the rectangular array of the possible consecutive integer series related to the integer fractions defining the $V_{F}$ overtones, $V_{F o}$ green integers referenced to as $\mathrm{n}_{1}$, the wavelengths of the overtones, $\lambda_{o}$ the green harmonic fractions, $1 / n_{2}$, the wavelengths of the partial harmonic fractions, $\lambda_{p s}$ the yellow fractions, $1-\left(1 / n_{2}\right)$, and the coupling constants of the partial harmonic fractions shaded in gray or blue of a classic harmonic standing wave $v_{F}$ system. The blue coupling constants are the related to transforming the smaller partial harmonic fraction to the larger. The gray coupling constants convert the larger partial harmonic fraction to the smaller. The fractions are a highly ordered system where all of the wavelengths, frequencies, and coupling constants of the possible harmonics are based on a single $v_{F}$ and the harmonic fraction series.

\begin{tabular}{|c|c|c|c|c|c|c|c|c|c|c|}
\hline$n_{1}$ & & 1 & 2 & 3 & 4 & 5 & 6 & 7 & \multicolumn{2}{|l|}{8} \\
\hline \multicolumn{11}{|c|}{$n_{2}$} \\
\hline \multirow{2}{*}{2} & 1 & 1 & 4 & 3 & 8 & 5 & 12 & 7 & 16 & \\
\hline & $\overline{2}$ & $\overline{2}$ & $\overline{3}$ & $\overline{2}$ & $\overline{5}$ & $\overline{3}$ & $\overline{7}$ & $\overline{4}$ & $\overline{9}$ & \\
\hline \multirow{2}{*}{3} & $\frac{1}{1}$ & $\underline{3}$ & 2 & 9 & 6 & 5 & 9 & 21 & 4 & \\
\hline & 3 & $\overline{4}$ & $\overline{3}$ & $\overline{8}$ & $\overline{5}$ & $\overline{4}$ & $\overline{7}$ & $\overline{16}$ & $\overline{3}$ & \\
\hline \multirow{2}{*}{4} & $\frac{1}{1}$ & $\underline{2}$ & 8 & 3 & 16 & 10 & 8 & 7 & 32 & \\
\hline & 4 & $\overline{3}$ & $\overline{9}$ & $\overline{4}$ & $\overline{15}$ & $\overline{9}$ & $\overline{7}$ & $\overline{6}$ & $\overline{27}$ & \\
\hline \multirow{2}{*}{5} & $\frac{1}{1}$ & $\underline{5}$ & $\underline{5}$ & 15 & $\underline{4}$ & 25 & 15 & 35 & 10 & \\
\hline & $\overline{5}$ & $\overline{8}$ & $\overline{6}$ & $\overline{16}$ & $\overline{5}$ & $\overline{24}$ & $\overline{14}$ & $\overline{32}$ & $\overline{9}$ & \\
\hline \multirow{2}{*}{6} & $\frac{1}{1}$ & $\underline{3}$ & 4 & 9 & 24 & 5 & 36 & 21 & 48 & \\
\hline & $\overline{6}$ & $\overline{5}$ & $\overline{5}$ & $\overline{10}$ & $\overline{25}$ & $\overline{6}$ & $\overline{35}$ & $\overline{20}$ & $\overline{45}$ & \\
\hline \multirow{2}{*}{7} & $\frac{1}{-1}$ & 7 & $\underline{7}$ & $\underline{7}$ & 14 & 35 & 6 & 49 & 28 & \\
\hline & 7 & $\overline{12}$ & $\overline{9}$ & $\overline{8}$ & $\overline{15}$ & $\overline{36}$ & $\overline{7}$ & $\overline{48}$ & $\overline{27}$ & \\
\hline \multirow{2}{*}{8} & $\frac{1}{1}$ & $\underline{4}$ & 16 & $\underline{6}$ & 32 & 20 & 48 & 7 & 64 & \\
\hline & $\overline{8}$ & $\overline{7}$ & $\overline{21}$ & $\overline{7}$ & $\overline{35}$ & $\overline{21}$ & $\overline{49}$ & $\overline{8}$ & 63 & \\
\hline \multirow{2}{*}{9} & $\frac{1}{1}$ & 9 & 3 & 27 & 9 & 45 & 27 & 63 & 8 & \\
\hline & $\overline{9}$ & $\overline{16}$ & $\overline{4}$ & $\overline{32}$ & $\overline{10}$ & $\overline{48}$ & $\overline{28}$ & $\overline{64}$ & $\overline{9}$ & \\
\hline$n_{2}$ & 1 & & & & & & & & & $1-\frac{1}{2}$ \\
\hline$\pi_{2}$ & $n_{2}$ & & & & & & & & & \\
\hline
\end{tabular}


the superposition interference of two waves of different frequency. The beat frequency is both a virtual frequency that can be mathematically derived and a real frequency that can be observed and measured in certain settings. A beat is essential to accurately define the actual physical interaction, superposition, of two different frequency waves. We define a beat frequency function $v_{b}\left(V_{1}, V_{2}\right)$ for frequencies $V_{1}, V_{2}$, where $V_{1}>V_{2}$ equaling their difference divided by 2, Equation (2). This relationship can also be defined as a ratio or coupling constant, $V_{2} / V_{1}$, Equation (2).

$$
v_{b}\left(v_{1}, v_{2}\right)=\left(\frac{v_{1}-v_{2}}{2}\right)=\frac{v_{1}}{2}\left(1-\frac{v_{2}}{v_{1}}\right)
$$

\subsection{Resonance of Two Wave Systems}

Resonance is another wave interaction of the likelihood of transfer of energy between two storage modes from one harmonic system to another. It is also the foundation of natural harmonics. The ratio of the frequencies defines the degree of possible resonance of the two waves. Therefore, the coupling constant of two frequencies mathematically encodes the beat, resonance, and many other wave interactions. Coupling constants physically, mathematically, and conceptually are related to the transformation of one entity or scalar to another.

\subsection{Dimensional Analysis of the Pythagorean Comma}

The musical system is analyzed as a dimensionless system, but in spontaneous physical systems, the units need to be accurately accounted. Dimensional analysis is the analysis of physical systems between different physical quantities focusing on their physical units [8] [9]. Equation (3) is equivalent to Equation (2) utilizing dimensional analysis methods for the Pythagorean Comma system, and a single unit of frequency. In this case, the physical units of $\mathrm{Hz}$ or $s$ are accurately maintained. A virtual harmonic frequency is essential to sustain true unit powers. We define a virtual frequency Pythagorean harmonic, $V_{P h}$, inter-relating the harmonic powers of 3 and 2 as $0.998066 \mathrm{~Hz}$. This frequency is referred to as a generalized virtual Pythagorean harmonic, $V_{P h}$ for any group of frequencies defined within a power law. For a homogenously dimensioned system the sum of the Hertzian powers of the higher frequency and the $V_{P h}$ equals the Hertzian power of the lower frequency.

$$
v_{P h}\left(3^{12}, 2^{19}\right)=(0.99806601 \mathrm{~Hz})=\left(\frac{2^{19}}{3^{12}}\right)^{1 / 7}
$$

\section{Materials and Methods}

\subsection{The Pythagorean Comma, The Buckingham Pi Theorem, Dimensional Analysis Connection}

The recognition of these harmonic power properties and the derivation of a virtual harmonic frequency for the Pythagorean Comma represents the first exam- 
ple of a parallel utilization of the Buckingham $\Pi$ theorem and dimensional analysis [8] [9]. It also represents an early example of the concept of logarithms. This generalized method was not described for millennia.

The Buckingham $\Pi$ theorem and dimensional analysis state that any individual physical unit is not invariant or essential. What are both invariant and fundamental are the coupling constants of two physical constants defined by the same physical unit, and ratios of their exponents based on a $V_{F}$ or the ratio of their logs. The standard physical unit of these ratios is irrelevant and could be a distance, frequency, mass, or energy. These methods reduce a complicated physical problem to the most straightforward perspective by utilizing the concepts of equivalence, unit transformation, reduction in the number of independent variables, by identifying dimensionless groups, relative magnitudes, and both base and derived quantities.

The independence of formulas from any specific unit, invariance of dimensionless ratios, and physical relationships is defined as the product of multiple entities raised to powers, and homogenous dimensions. In Equation (4) the standard formula of a Buckingham-derived quantity, $\Pi_{i}$, equals the product of powers of the independent variables $Q_{n}$ defining a specific physical property. The powers are usually derived by inspection, intuition, or more complicated algebraic methods. A power law can be calculated even when the physical relationships are not explicitly known. For example, if all of the terms for a physical system are known then the powers can be derived with a computer multi-dimensional search power engine when their power ratios equal unity or the closest to unity [10] [11] [12].

$$
\left[\Pi_{i}\right]=\left[Q_{1}^{n_{1}} Q_{2}^{n_{2}} \cdots Q_{k}^{n_{k}}\right]
$$

We analyze the generalized physical setting of a Pythagorean power law harmonic related to the maximum power resonance for two frequencies, Equations $((5),(6))$. There are two different frequencies $v_{1}$ and $v_{2}$, and are both higher than 1 , and by our convention, $V_{1}$ is greater than $V_{2}$. Their respective Pythagorean harmonic powers are $n_{1}$ and $n_{2}$; where $n_{2}$ must be larger than $n_{1}$. Table 2 is an integer fraction array of the possible powers of all of the Pythagorean power laws for two frequencies. Table $2 \&$ Table 3 are the integer fraction arrays of the powers, coupling constants, and the infinite number of frequencies of the Pythagorean power law harmonics related to a $V_{F}$ analogous to a standing wave system. For any two individual frequencies there are an infinite number of cells from the array will represent that specific Pythagorean power law. The ratio of the powers is constant not the individual powers. The smallest powers are those defined. The multiples also fulfill this requirement. From dimensional analysis, the sum of the dimensional exponents of $V_{1}$, i.e. $\mathrm{n}_{1}$, and $V_{P h}$, i.e. $n_{2}-n_{1}$, must equal $n_{2}$. The specific powers associated with the dimensionless ratio are those powers of the frequencies closest or equal to dimensionless 1 and $V_{P h}$ closest or equal to $1 \mathrm{~Hz}$ that repeat at integer power multiples. This leads to a specific solution for the lowest powers for each pair of frequencies. As the powers increase 
Table 2. Pythagorean possible power matrix for two specific frequencies. This table is the rectangular array of the possible consecutive integer series powers for a Pythagorean power law of a system $V_{P h}$ equal to or nearly to $1 \mathrm{~Hz}$. Here $n_{1}$ are the powers for $V_{1}$, and $n_{2}$ are the powers of $V_{2}$. For two frequencies there are an infinite number of cell possibilities with $V_{P h}$ closest or equal to $1 \mathrm{~Hz}$. The ratio of $n_{1} / n_{2}$ is constant not the individual powers. The cells associated with the $V_{P h}$ do have a repeating pattern based on the consecutive integer series. The cells labeled with * are examples of first two of an infinite number of repeating powers with the same $V_{P h}$. The frequency of $v_{2}$ is listed above, and the Pythagorean power law listed below with their $\mathrm{Hz}$ or time units in each cell. The Pythagorean power law is shown as the product of one of the frequencies and a power of the $V_{2} / V_{1}$ coupling constant. The diagonal where $n_{1}$ equals $n_{2}$ are the powers of their coupling constant highlighted in blue. The cells with units of $H z$ are highlighted in yellow and are all partial harmonic values.

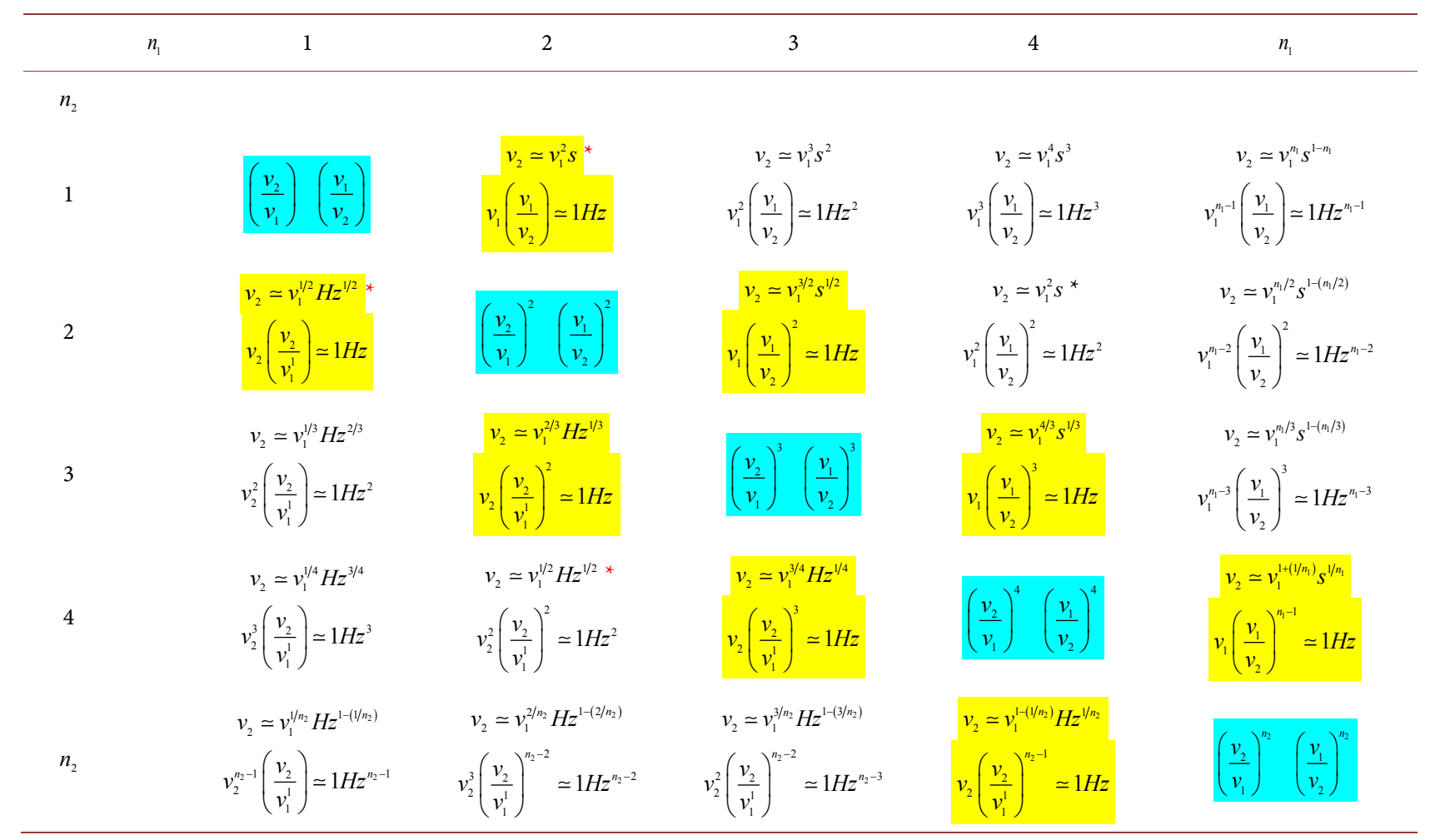

the pattern repeats and the identical $V_{P h}$ returns. A second alternate, but equivalent mathematical and physical definition is $V_{P h}$ divided by either frequency raised to the $n_{2}-n_{1}$ power equals the coupling constant of the two frequencies raised to a power of $n_{2}$ or $n_{1}$, Equation (6). The third alternative is virtual harmonic frequency equals the product of one frequency to a power and their coupling constant raised to a power, Equation (6). The fourth alternative is one frequency equals the product of the other frequency and the $V_{P h}$ raised to powers.

$$
\begin{gathered}
{\left[v_{P h}\left(v_{1}^{n_{1}}, v_{2}^{n_{2}}\right) H z\right]^{\left(n_{2}-n_{1}\right)}\left[v_{1} H z\right]^{n_{1}}=\left[v_{2} H z\right]^{n_{2}}} \\
v_{P h}\left(v_{1}^{n_{1}}, v_{2}^{n_{2}}\right)=\left(\frac{v_{2}^{n_{2}}}{v_{1}^{n_{1}}}\right)^{1 /\left(n_{2}-n_{1}\right)}=v_{1}\left(\frac{v_{2}}{v_{1}}\right)^{n_{2} /\left(n_{2}-n_{1}\right)}=v_{2}\left(\frac{v_{2}}{v_{1}}\right)^{n_{1} /\left(n_{2}-n_{1}\right)}
\end{gathered}
$$

These are many algebraic isomorphic transformations possible, Equations ((5), (6)). The notation for the Pythagorean power harmonic of two frequencies is $v_{P h}\left(v_{1}^{n_{1}}, v_{2}^{n_{2}}\right)$. We utilize functional notation of the higher frequency and its power followed by the lesser frequency, and its power in parentheses. 
Table 3. Pythagorean power matrix for a fundamental frequency and its infinite number of possible harmonics. This table is the rectangular array of the possible consecutive integer series powers for a Pythagorean power law of a $V_{F}$ system where all the $V_{P h}$ are equal to $1 \mathrm{~Hz}$. All of the possible harmonics, $V_{2}$, are based on powers of $V_{F}$. This array is structured similarly to Table 1 and the standing wave system, but for a Pythagorean power law system. $n_{1}$ are the power possibilities of $V_{F}$ which equals $V_{1}$, and $n_{2}$ are the power possibilities of $v_{2}$. The frequency of $v_{2}$ as a power of $v_{F}$ is listed above, and the Pythagorean power law listed below with their time units, $\mathrm{Hz}$ or $s$, in each cell. There are five sets of harmonics specifically associated with the harmonic fraction system of a standing wave, Table 1. The series highlighted blue diagonal set are all related to dimensionless coupling constants transforming $v_{F}$ to the cells from the one on the right to the left. These are related to the dimensionless $V_{F} s$ raised to the \pm harmonic fraction series. The horizontal set highlighted in green are related to $v_{F}^{n_{1}}$, and corresponds the harmonic overtone series. The vertical green highlighted set are all related to $v_{F}^{1 / n_{2}}$, and correspond to the wavelengths of the overtones. The yellow diagonal sets below and above the coupling constant set are related to the partial harmonic fractions $1 \pm\left(1 / n_{2}\right)$. These represent valid possible physical frequencies since they have $\mathrm{Hz}$ or $s$ units. The unshaded cells are those with powers of $\mathrm{Hz}$ greater than 1 . This number pattern corresponds to the classic harmonic number set, Table 1 . The cells repeat as seen with the cells labeled with an asterisk.

\begin{tabular}{|c|c|c|c|c|c|}
\hline$n$ & 1 & 2 & 3 & 4 & $n_{1}$ \\
\hline \multicolumn{6}{|l|}{$n_{2}$} \\
\hline \multirow[b]{2}{*}{1} & & $v_{2}=v_{F}^{2} s^{*}$ & $v_{2}=v_{F}^{3} s^{2}$ & $v_{2}=v_{F}^{4} s^{3}$ & $v_{2}=v_{F}^{n_{1}} S^{n_{1}-1}$ \\
\hline & $\left(\frac{v_{2}}{v_{F}}\right)$ or $\left(\frac{v_{F}}{v_{2}}\right)$ & $\frac{v_{F}^{2}}{v_{2}}=1 H z$ & $\frac{v_{F}^{3}}{v_{2}}=1 H z^{2}$ & $\frac{v_{F}^{4}}{v_{2}}=1 H z^{3}$ & $\frac{v_{F}^{n_{1}}}{v_{2}}=1 H z^{n_{1}-1}$ \\
\hline \multirow[b]{2}{*}{2} & $v_{2}=v_{F}^{1 / 2} H z^{1 / 2} *$ & & $v_{2}=v_{F}^{3 / 2} s^{1 / 2}$ & $v_{2}=v_{F}^{2} s^{*}$ & $v_{2}=v_{F}^{n_{n} / 2} S^{\left(n_{1} / 2\right)-1}$ \\
\hline & $\frac{v_{2}^{2}}{v_{F}}=1 \mathrm{~Hz}$ & $v_{F}^{ \pm 1 / 2} S^{ \pm 1 / 2}$ & $\frac{v_{F}^{3}}{v_{2}^{2}}=1 H z$ & $\frac{v_{F}^{4}}{v_{2}^{2}}=1 H z^{2}$ & $\frac{v_{F}^{n_{1}}}{v_{2}^{2}}=1 H z^{n_{1}-2}$ \\
\hline \multirow[b]{2}{*}{3} & $v_{2}=v_{F}^{1 / 3} H z^{2 / 3}$ & $v_{2}=v_{F}^{2 / 3} H z^{1 / 3}$ & & $v_{2}=v_{F}^{4 / 3} s^{1 / 3}$ & $v_{2}=v_{F}^{n_{1} / 3} S^{\left(m_{1} / 3\right)-1}$ \\
\hline & $\frac{v_{2}^{3}}{v_{F}}=1 H$ & $\frac{v_{2}^{3}}{v_{F}^{2}}=1 \mathrm{~Hz}$ & $v_{F}^{ \pm 1 / 3} s^{ \pm 1 / 3}$ & $\frac{v_{F}^{4}}{v_{2}^{3}}=1 H z$ & $\frac{v_{F}^{n_{1}}}{v_{2}^{3}}=1 H z^{n_{1}-3}$ \\
\hline \multirow[b]{2}{*}{4} & $v_{2}=v_{F}^{1 / 4} H z^{3 / 4}$ & $v_{2}=v_{F}^{1 / 2} H z^{1 / 2} \star$ & $v_{2}=v_{F}^{3 / 4} H z^{1 / 4}$ & & $v_{2}=v_{F}^{1+\left(1 / n_{1}\right)} S^{1 / n_{1}}$ \\
\hline & $\frac{v_{2}^{4}}{v_{F}}=1$ & $\frac{v_{2}^{4}}{v_{F}^{2}}=1 H z^{2}$ & $\frac{v_{2}^{4}}{v_{F}^{3}}=1 H z$ & $v_{F}^{ \pm 1 / 4} s^{ \pm 1 / 4}$ & $\frac{v_{F}^{n_{1}}}{v_{2}^{n_{1}-1}}=1 H z$ \\
\hline & $v_{2}=v_{F}^{1 / n_{2}} H z^{1-\left(1 / n_{2}\right)}$ & $v_{2}=v_{F}^{2 / n_{2}} H z^{1-\left(2 / n_{2}\right)}$ & $v_{2}=v_{F}^{3 / n_{2}} H z^{1-\left(3 / n_{2}\right)}$ & $v_{2}=v_{F}^{1-\left(1 / n_{2}\right)} H z^{1 / n_{2}}$ & \\
\hline$n_{2}$ & $\frac{v_{2}^{n_{2}}}{v_{F}}=1$ & $\frac{v_{2}^{n_{2}}}{v_{F}^{2}}=1 H z^{n_{2}-2}$ & $\frac{v_{2}^{n_{2}}}{v_{F}^{3}}=1 H z^{n_{2}-3}$ & $\frac{v_{2}^{n_{2}}}{v_{F}^{n_{2}-1}}=1 H z$ & $v_{F}^{ \pm 1 / n} S^{ \pm 1 / n}$ \\
\hline
\end{tabular}

We developed a generalized computer multi-dimensional matrix search engine utilizing MATLAB that derives the Pythagorean power law through a number search starting with the smallest integers, using Equations ((5), (6)) [10] [11] [12]. This methodology is valid based on searching through a consecutive integer series. The pattern repeats when the power ratio repeats.

If the power ratio of two frequencies equals unity, then $V_{P h}$ equals $1 \mathrm{~Hz}$. In this setting one frequency is a power of the other. If there is a $V_{F}$ all other possible frequencies are mathematically related through power laws, and all $V_{P h}$ equal 1 
$\mathrm{Hz}$, Table 3. The ratio of the logs of the frequencies and the powers equals $n_{1} / n_{2}$, and are rational. The $\log$ of $1 \mathrm{~Hz}$ is 0 and rational. The power of $V_{P h}$ is 0 rather than the $v_{b}$ equaling $0 \mathrm{~Hz}$ in the standard beat relationship. If there is no power of $V_{1}$ that equals the power of $V_{2}$ then the $V_{P h}$ will not be equal to $1 \mathrm{~Hz}$. It is irrational, and the ratio of the logs of the frequencies will not be rational.

The $v_{P h}\left(v_{1}^{n_{1}}, v_{2}^{n_{2}}\right)$, is specific for two frequencies, $v_{1}, v_{2}$, with a specific ratio of powers, $n_{1} / n_{2}$. Here $\mathrm{n}_{1}$ and $\mathrm{n}_{2}$ are the lowest values. There is an infinite number of other $n_{1} / n_{2}$ possibilities as the $n^{\prime}$ multiples increase. The $\left(n^{\prime} n_{1}\right) /\left(n^{\prime} n_{2}\right)$ pair keeps repeating as each reaches the next integer multiple possibilities. The ratio of the logs of $V_{1}$ and $V_{2}$ are equal to or nearly equal to the ratio of the powers. Any power law of these two frequencies must be related to $V_{P h}$ independently even if they are not associated with the maximum resonant $v_{P h}$ state. Either frequency, $V_{1}$ or $V_{2}$, can represent a power base frequency. All products of either frequency and any power of their coupling constant are part of this harmonic system. Defining one frequency as the power of another is valid and is based on the dimensionally homogenous relationships, Equations ((5), (6)). Defining one frequency as the power of another is not typically done within standard physics.

\subsection{The Coupling Constant Perspective}

There are five dimensionless algebraic isomorphic equivalents of Equations ((5), (6)) viewed as coupling constants, including $V_{P h}$, Equations (7)-(9). Coupling constants are a critical aspect of this harmonic system and explain why they can be viewed simultaneously as homogeneously dimensioned or as dimensionless relationships. This coupling constant property is a characteristic of both the Buckingham and dimensional analysis methods. The coupling constant of $V_{2}$ divided by $V_{1}$ equals 1 minus the ratio of twice $V_{b}\left(V_{1}, V_{2}\right)$ divided by $V_{1}$. The coupling constant of $V_{2}$ divided by $V_{1}$ also equals the coupling constant of $V_{P h}$ divided by $V_{1}$ raised to the power $\left(n_{2}-n_{1}\right) / n_{2}$, or the coupling constant of $V_{P h}$ divided by $V_{2}$ raised to $\left(n_{2}-n_{1}\right) / n_{1}$. In Equation (8) dimensionless $V_{1}$ s raised to the exponent $\left(n_{1} / n_{2}-1\right)$ plus the product $\left(n_{2}-n_{1}\right) / n_{2}$ and $\log \left(V_{P h}\right) / \log \left(V_{1}\right)$. Here $\log \left(v_{P h}\right) / \log (v)$ is equivalent to $\log _{v}\left(v_{P h}\right)$, the log base $v$. In Equation (9) dimensionless $V_{2} \mathrm{~s}$ raised to the exponent $\left(n_{2} / n_{1}-1\right)$ minus the product $\left(n_{2}-n_{1}\right) / n_{1}$ and $\log \left(V_{P h}\right) / \log \left(v_{2}\right)$. These are isomorphic relations. The coupling constants of the Pythagorean power laws are dependent on the base $v$. The coupling constants for a standing wave system do not change.

$$
\begin{gathered}
\frac{v_{2}}{v_{1}}=1-\left(\frac{2 v_{b}\left(v_{1}, v_{2}\right)}{v_{1}}\right)=\left(\frac{v_{P h}\left(v_{1}^{n_{1}}, v_{2}^{n_{2}}\right)}{v_{2}}\right)^{\frac{\left(n_{2}-n_{1}\right)}{n_{1}}}=\left(\frac{v_{P h}\left(v_{1}^{n_{1}}, v_{2}^{n_{2}}\right)}{v_{1}}\right)^{\frac{\left(n_{2}-n_{1}\right)}{n_{2}}} \\
\frac{v_{2}}{v_{1}}=\left(v_{1} s\right)^{\left(\left(n_{1} / n_{2}\right)-1\right)+\left(\left(n_{2}-n_{1}\right) / n_{2}\right)\left(\log \left(v_{P h} s\right) / \log \left(v_{1} s\right)\right)} \\
\frac{v_{1}}{v_{2}}=\left(v_{2} s\right)^{\left(\left(n_{2} / n_{1}\right)-1\right)-\left(\left(n_{2}-n_{1}\right) / n_{1}\right)\left(\log \left(v_{P h} s\right) / \log \left(v_{2} s\right)\right)}
\end{gathered}
$$




\subsection{The Log Coupling Constant Perspective}

The coupling constant ratios of the logs of the two frequencies are invariant and equal or nearly equals the ratios of $n_{1}$ and $n_{2}$, Equations ((10), (11)). The ratio of the logs is a unique component of the Pythagorean system. Transformation to other units than $\mathrm{Hz}$ is related to Equations ((10), (11)) multiplied and divided by the same transformational constant. Conversion to other units is analogous to different keys in music, where the harmonic structure and powers are unaltered.

$$
\begin{aligned}
& \frac{\log \left(v_{2} s\right)}{\log \left(v_{1} s\right)}=\log _{v_{1}}\left(v_{2} s\right)=\frac{n_{1}}{n_{2}}+\left(\frac{n_{2}-n_{1}}{n_{2}}\right)\left(\log \left(v_{P h} s\right) / \log \left(v_{1} s\right)\right) \\
& \frac{\log \left(v_{1} s\right)}{\log \left(v_{2} s\right)}=\log _{v_{2}}\left(v_{1} s\right)=\frac{n_{2}}{n_{1}}-\left(\frac{n_{2}-n_{1}}{n_{1}}\right)\left(\log \left(v_{P h} s\right) / \log \left(v_{2} s\right)\right)
\end{aligned}
$$

\subsection{The Essential Physical Meaning of a Pythagorean Power Harmonic and Its Relationship to the Beat}

The $V_{P h}$ is an essential physical constant that maintains homogenous units even if the scalar value is 1 . When the $V_{P h}$ is not equal to $1 \mathrm{~Hz}$, then there will be a beat associated with the two most resonant frequencies. This beat may be measurable and can be utilized to derive the $V_{P h}$ as in Equations ((12), (13)). Equations ((12), (13)) demonstrate that the $v_{b}$ is derived from the $v_{P h}$, or visa versa.

$$
\begin{aligned}
& v_{b}\left(v_{1}, v_{2}\right)=\frac{v_{1}}{2}\left(1-\left(\frac{v_{P h}\left(v_{1}^{n_{1}}, v_{2}^{n_{2}}\right)}{v_{1}}\right){ }^{\frac{\left(n_{2}-n_{1}\right)}{n_{2}}}\right) \\
& v_{P h}\left(v_{1}^{n_{1}}, v_{2}^{n_{2}}\right)=v_{1}\left(1-\left(\frac{2 v_{b}\left(v_{1}, v_{2}\right)}{v_{1}}\right)\right)^{n_{2} /\left(n_{2}-n_{1}\right)}
\end{aligned}
$$

\section{Results}

\subsection{The Generalized Pythagorean Power Laws Are Isomorphic with the Coupling Constants and the Beat}

Equations (7)-(9) demonstrate that the coupling constants are encoded in an infinite number of isomorphic Pythagorean power laws. The beat is also defined within the power laws, Equations ((12), (13)).

\subsection{The Coupling Constant Ratio of the Log of the Frequencies Nearly or Equals the Ratio of the Powers}

Equations (10) and (11) show that the ratio of the powers of the Pythagorean power law are related to the ratio of the logs of the frequencies. The log ratio can be a close estimate or exact value of the ratio of the powers. The difference of the ratio of the logs of the frequencies minus the ratio of the powers equals a function of the product of $n_{1}$ and $n_{2}$ and the $\log$ of $V_{P h}$. It is another fundamental constant of the system. For a system based on a $V_{F}$ the powers associated with $\mathrm{Hz}$ 
units must equal $n_{2}-n_{1}$ equals 1 or $n_{1}-n_{2}$ equals 1 because they are partial harmonic fractions, Table $2 \&$ Table 3 . The partial harmonic fractions are natural harmonics based on their unit.

\subsection{The Generalized Pythagorean Powers of a Fundamental Frequency Are in a Form Identical to the Harmonic of a Standing Wave System}

There is an infinite number of power harmonics of $V_{F}$ and $V_{2}$ related to $V_{F}$ raised to powers. Table 1 is the matrix array of the harmonic standing wave system of the overtones, $o t$, harmonic fractions, $1 / n$, partial harmonic fractions, $1 \pm(1 / n)$, and the coupling constants, $\left(n_{1}^{\prime} / n_{2}^{\prime}\right)\left(n_{2}^{\prime \prime} / n_{1}^{\prime \prime}\right)$, transforming any partial harmonic fraction to another. If a $V_{F}$ defines all of the possible harmonics, then all of the $V_{P h}$ must equals $1 \mathrm{~Hz}$. Table 3 is the matrix array of the possible powers of a $V_{F}$ system. There are five portions of the array that are related to the structure of standing wave harmonics. $v_{F}^{n} s^{n-1}$ is equivalent to the overtone series. $v_{F}^{1 / n} H z^{1-(1 / n)}$ or $\left(v_{F} s\right)^{1 / n}$ are associated with the harmonic fraction series and both the wavelengths and the coupling constants. Those harmonics defined by the physically valid unit $H z$ to the first power $v_{F}^{1-(1 / n)} H z^{1 / n}$ and $v_{F}^{1+(1 / n)} s^{1 / n}$ are related to the partial harmonic fractions. Both the Pythagorean and the standing wave harmonic systems have the same matrix fraction system, Table 1 \& Table 3.

\section{Discussion}

\subsection{Fusion of the Harmonic Power Laws of Wave Interactions Creates a New Fourier Perspective}

These ancient observations represent a profound conceptual, physical, mathematical, and harmonic insight. They encode the interaction between two different frequency waves into a power law relationship that encodes all other possible harmonic wave interactions and encodes explicitly their coupling constant. The beat does not encode the coupling constant, but both are essential to describe the interactions of two waves entirely. Power laws and coupling constants are ubiquitous in physics and discrete probabilities. Despite its absence in the literature, a generalized Pythagorean harmonic power perspective represents a valid, inventive, parallel approach to analyzing wave systems. Instead of the standard 1D Fourier linear perspective, this is a $2 \mathrm{D}$ natural unit log or exponent Fourier approach. The second dimension is related to the powers of $V_{P h}$. The natural units are the frequencies of $V_{1}$ and $V_{2}$. They represent classic inverse mathematical transformations from linear to power relationships, and as such constitute an isomorphic system.

\subsection{The Significance}

This generalized Pythagorean power law perspective is important since it is a "new" method to interrogate and evaluate harmonic systems. Harmonic systems 
outside of music have not been evaluated with the method. There is new harmonic information encoded in the Pythagorean power laws not in the standard physical methods. The virtual harmonic of a $v_{F}$ system is always linked to $1 \mathrm{~Hz}$ which is the wavelength of the fundamental just like the standing wave system. Though the Pythagorean law is related to two frequencies a system with any number of frequencies can be evaluated as well. The same methods can be utilized. In physical systems the power harmonics would always be the closest to 1 Hz. It is remarkable that a $V_{F}$ system for those defined by a consecutive integer series is identical to that of the standard harmonic systems. Therefore any system based on a fundamental frequency will demonstrate simultaneous mathematical and physical harmonic number properties in the standard time domain and in the power law domain. This is known from music and sonic systems, but has not been described or observed in spontaneous physical harmonic systems. Without evaluation from the Pythagorean law perspective these relationships will not be recognizable. It is a repeating occurrence in science that physical systems are discovered to be defined within mathematical systems that initially appear to have no broad physical counterpart [10] [11] [12]. The Pythagorean power harmonic system is associated with an infinite number of possible powers. This is a characteristic of quantum systems. It is not part of the standing wave where there is a repeating constant harmonic as the powers proceed towards infinity.

\section{Conclusions}

\subsection{New Perspective on Wave Interactions}

The generalized Pythagorean power relationship is a new perspective on the mathematics and physics of unified harmonic systems based on ancient valid physical and mathematical concepts described by Pythagoras and other Greeks. The power of the Greeks imagination and intellect is shocking since their observations describe a virtual power harmonic encoding the coupling of two waves preceding the recognition of a beat and interference. This harmonic system represents a known physical phenomenon and is an isomorphic mathematical representation of wave properties.

\subsection{New Future Insights into Actual Physical Systems}

This Pythagorean power perspective will generate many valuable insights into the structure of natural, spontaneous harmonic systems that are not even suspected. The Pythagorean power system have mathematical properties that parallel quantum systems where there are an infinite number of possibilities, but when analyzed only generate a single recurring result.

\section{Acknowledgements}

I want to thank Thomas Budinger PhD, Vola Andrianarijaona PhD, and Richard Vento MS for their insights, advice, and help. 


\section{References}

[1] Wright, D. (2009) Mathematics and Music, Mathematical World. American Mathematical Society. Providence, Rhode Island, 28, 53-59, 129-134.

[2] Durfee, D. and Colton, J. (2015) The Physics of Musical Scales: Theory and Experiment. American Journal of Physics, 83, 835. https://doi.org/10.1119/1.4926956

[3] Jeans, J. (1968) Science and Music. Dover, 154.

[4] Katznelson, Y. (2004) An Introduction to Harmonic Analysis. 3rd Edition, Cambridge University Press, Cambridge. https://doi.org/10.1017/CBO9781139165372

[5] Schroeder, M. (1991) Fractals, Chaos, Power Laws Minutes from an Infinite Paradise. Dover Publication Incorporated, Mineola.

[6] Schopenhauer, A. (1966) The World as Will and Representation. Dover Publications, E.F.J. Payne translation, 1, 266.

[7] Antoine, P., L'Huillier, A. and Lewenstein, M. (1996) Attosecond Pulse Trains Using High-Order Harmonics. Physical Review Letters, 77, 1234. https://doi.org/10.1103/PhysRevLett.77.1234

[8] Brand, L. (1957) The Pi Theorem of Dimensional Analysis. Archive for Rational Mechanics and Analysis, 1, 35-45. https://doi.org/10.1007/BF00297994

[9] Saichev, A., Malevergne Y. and Sornette, D. (2009) Theory of Zipf's Law and Beyond. Lecture Notes in Economics and Mathematical Systems, 632.

[10] Chakeres, D., Buckhanan, W. and Andrianarijaona, V. (2017) Systematic Harmonic Power Laws Inter-Relating Multiple Fundamental Constants. American Physical Society April Meeting, Washington DC.

[11] Chakeres, D. (2018) The Pythagorean Comma-Buckingham $\Pi$ Theorem Perspective on the Inter-Relationship of the Hydrogen Quanta. American Physical Society, Los Angeles.

[12] Chakeres, D. (2018) Pythagorean Comma, Buckingham Pi Theorem, Dimensional Analysis, and Harmonic Power Laws of the Frequency Equivalents of the Electron, Bohr Radius, and Rydberg Constant. American Physical Society, Columbus. 